\title{
The Influence of Maximum Strength Performance in Squats and Standing Calf Raises on Squat Jumps, Drop Jumps, and Linear as well as Change of Direction Sprint Performance in Youth Soccer Players
}

\author{
Michael Keiner $^{1 *}$, Björn Kadlubowski ${ }^{1,2}$, Hagen Hartmann ${ }^{1}$, Tobias Stefer ${ }^{1}$ and Klaus Wirth ${ }^{3}$ \\ ${ }^{1}$ German University of Health \& Sport, Germany \\ ${ }^{2}$ DSC Arminia Bielefeld e. V, Germany
}

${ }^{3}$ University of Applied Sciences Wiener Neustadt, Austria

*Corresponding author: Michael Keiner, German University of Health \& Sport, Steinheilstraße 4, 85737 Ismaning, Germany,E-mail: michaelkeiner@gmx.de

\begin{abstract}
Background: Speed and strength performance have an essential role in soccer. It is well known there is a moderate to strong correlation between maximum strength, speed and jump performance. However most studies have analyzed only maximum strength values of the knee and hip extensors for the correlation analysis. Studies on the maximum strength of the calf muscles are rare.

Methods: The aim of the study was to calculate the onetailed correlation coefficients of the One-Repetition Maximum (1RM) in standing calf raises (SCR) and squat performance on linear (LS, $20 \mathrm{~m}$ ) and change of direction sprints (CODs, $10 \mathrm{~m}, 2$ turns), squat jumps (SJs) and drop jumps (DJs) from heights of $24 \mathrm{~cm}, 32 \mathrm{~cm}$ and $40 \mathrm{~cm}$ in youth soccer players $(n=19,16.5 \pm 0.5$ years old $)$.

Results: The results of this study show weak to strong correlations of absolute $\left(r^{2}=0.21-0.49\right)$ and relative to body weight back squat $\left(r^{2}=0.01-0.13\right)$ performance with SJ, LS and COD performance. The absolute and relative strength in SCR was lower in these variables $\left(r^{2}=0.01-0.13\right)$. DJ performance is independent of DJ height and can be explained to a greater extent via the relative to body weight $1 \mathrm{RM}$ in $\operatorname{SCR}\left(r^{2}=0.17-0.32\right)$. Correlations of absolute and relative back squats to DJ performance were lower $\left(r^{2}=0.01-0.18\right)$. The data show that the 1RM for squats leads to a higher variance explanation for the variables LS, SJ and COD than the 1RM for SCR; however, the relative SCR, in particular, leads to higher explanation rates for DJ performance.
\end{abstract}

Conclusion: These results indicate that relative and absolute strength performance might have different impacts on jump, sprint and COD performance. Additionally, a limiting role of the triceps surae in DJ performance might be concluded.

\section{Keywords \\ Sports performance; Speed strength; Reactive strength}

\section{Background}

For match-play demands in team sports, players have to perform several high-intensity tasks during a game [1]. Because of changes in technical, tactical and physical requirements, soccer games have become more dynamic and fast paced $[2,3]$. Physiological factors, such as the capacity of soccer players to produce various forceful and explosive actions, are becoming increasingly essential to consider for players to exhibit optimal performance [4]. In addition to jumps, shots and linear sprints, athletes must also perform sprints with directional changes [5]. In general, a soccer player performs explosive actions every $2-4$ seconds and up to 1200-1400 explosive actions total during a game [6].

Physiologically, the maximum strength of a muscle depends on its cross-sectional area and neuronal acti-

Citation: Keiner M, Kadlubowski B, Hartmann H, Wirth K (2021) The Influence of Maximum Strength Performance in Squats and Standing Calf Raises on Squat Jumps, Drop Jumps, and Linear as well as Change of Direction Sprint Performance in Youth Soccer Players. Int J Sports Exerc Med 7:190. doi. org/10.23937/2469-5718/1510190

Accepted: May 03, 2021; Published: May 05, 2021

Copyright: (c) 2021 Keiner M, et al. This is an open-access article distributed under the terms of the Creative Commons Attribution License, which permits unrestricted use, distribution, and reproduction in any medium, provided the original author and source are credited. 
vation [7-9]. It is well known that there is mostly a moderate to strong correlation between maximum strength, speed and jump performance (linear sprints [LSs, distance 5 to $20 \mathrm{~m}$ ]: $\mathrm{r}^{2}=0.10-0.88$; squat jumps [SJs]: $\mathrm{r}^{2}=$ $0.58-0.60$; change of direction sprints [CODs]: $r^{2}=0.14$ $-0.40)[5,10-13]$. However, only a few studies have analyzed the correlation between the drop jump (DJ) and maximum strength performance. A previous study [14] determined that the jump height of the DJ of professional soccer players was significantly correlated with the player's dynamic maximal strength performance on a leg extensor machine $\left(r^{2}=0.19\right)$ and a leg curl machine $\left(r^{2}=0.22\right)$. However, there were only slight, nonsignificant correlations between these strength parameters and the ground contact time. Broich [14] concluded that the dynamic strength level of the knee flexors and extensors affects performance in fast (ground contact time $<250 \mathrm{~ms}$ [15]) stretch and shortening cycles (SSCs). In DJs that control for fast SSCs, there are small degrees of knee and hip flexion at ground contact. The stiffness of these muscles in the eccentric phase determines the neuronal response after the tendomuscular mechanism and can lead to short ground contact times and the development of forces exceeding those in the pure concentric phase [16]. The concentric forces generated are determined by the maximum strength [15], and the correlations presented by Broich [14] can therefore be explained logically. This finding was, in general, supported by data presented by Ferreira, et al. [17] and Dymond, et al. [18], who showed that the one-repetition maximum (1RM) for squats has a strong $\left(r^{2}=0.40-0.52\right)$ correlation with the DJ jump height; however, information about the ground contact time was missing from these studies. One recent study by Beattie, et al. [19] showed that there was a moderate $\left(r^{2}=0.09-0.16\right)$ between the isometric mid-thigh pull peak force and the reactive strength index (based on the ground contact time and jump height).

Most studies have analyzed only the maximum strength values of the knee and hip extensors for the correlation analysis of jump, sprint and COD performance. However, the musculus triceps surae plays an important role in sprint and jump performance. To the authors' knowledge, there are only two studies that have analyzed the correlation of maximum strength performance in SCR with jump or speed performance. Möck, et al. [20] showed medium to strong correlations in their study for both the absolute $\left(r^{2}-0.23\right.$ to $\left.r^{2}-0.52\right)$ and relative $\left(r^{2}-0.21\right.$ to $\left.r^{2}-0.32\right) 1 R M$ in SCR and the sprinting times for consecutive sections up to $30 \mathrm{~m}$. In their study, Hartmann, et al. [21] showed a correlation between the maximum SCR performance and the SJ $\left(r^{2}\right.$ $=0.44)$, CMJ $\left(r^{2}=0.49\right)$ and DJ $\left(r^{2}=0.13-0.33\right)$ performance.

Therefore, the aim of the study was to evaluate and compare the influence of the $1 \mathrm{RM}$ for SCRs and squats on LS, COD, SJ and DJ performance in elite youth soccer players. It was hypothesized that SCR and squat performance would have a moderate to strong correlation with LS, COD, SJ and DJ performance.

\section{Methods}

To answer the research question, 19 trained youth soccer players were recruited. They underwent maximum strength tests for the back squat (BS) 1RM BS, the standing calf raise with a barbell (1RM SCR), the SJ, drop jumps from heights of 24 centimeters $(\mathrm{cm}), 32 \mathrm{~cm}$ and $40 \mathrm{~cm}$ [DJ24, DJ32, DJ40, respectively]), the 20-m LS and COD. The tests were carried out on 2 test days separated by 4 days. On test day $1,20-\mathrm{mLS}$ and COD performance was analyzed first, followed by the 1 RM BS. On test day 2, jump performance followed by the 1RM SCR was assessed. One week before test day 1 , the soccer players completed a familiarization session for all tests on two separate days.

\section{Participants}

Nineteen male youth soccer players (height: 1.76; \pm $0.07 \mathrm{~m}$; weight: $62.5 \pm 8.6$ kilogram (kg); age: $16.5 \pm 0.5$ years old) were recruited from a training center for individuals under 17 years old (U17). The youth soccer players competed at the regional level. All participants had played soccer since their early childhood and were considered well trained relative to others of their age. The soccer players underwent performance tests as part of their semiannual performance diagnostics routine. The participants did not participate in fatiguing training sessions for a minimum of 3 days before testing. None of the participants reported any injuries at the time of testing.

Each participant and his parents were informed about the experimental risks involved with the research. All participants and their parents (if the participant was not 18 years old) provided written informed consent to participate in the present study. Furthermore, this study was approved by the institutional review board (German University of Health \& Sport, No. 01/2019.92002800). The study was performed with human participants in accordance with the Helsinki Declaration.

\section{Measures and procedures}

During testing, the $1 \mathrm{RMBS}$ (high bar) was determined. The barbell was positioned on the musculus trapezius pars descendens below the seventh cervical vertebra. The participants stood erect with a self-selected width of the feet, flexed their knees and hips to reach a deep squat position with proper form (top of thigh breaking parallel) and returned to the starting position. Attempts were considered failures when the participants rounded their backs or were not able to flex their knees to the desired depth. A warm-up (two sets of 6 to 8 reps) was performed with a submaximal, nonfatiguing load for the BS. The 1 RM BS was determined within a maximum of 5 trials. The rest duration between attempts was at least 
5 minutes $(\mathrm{min})$. The test-retest reliability, with an intraclass correlation coefficient (ICC) $=0.95-0.99$, was calculated in previous research $[22,23]$. In many sports, the body must move quickly during sprints and jumps. Therefore, strength performance should be evaluated in relation to body weight rather than in absolute values only. The maximum strength relative to body mass (REL BS) was calculated (REL BS $=1 \mathrm{RM} B S /$ body mass).

The maximum strength of the calf muscles was measured with the 1RM SCR. The participants stood erect with a self-selected foot width and the forefoot elevated by $2 \mathrm{~cm}$ (heels not elevated). The barbell was guided upwards along a metal extension of the rack. The heels were raised by $6 \mathrm{~cm}$ by extending the ankles, and the height was checked visually via a marker. Attempts were considered failures when the participants rounded their backs, flexed their knees or were not able to plantar flex their ankles to raise their heels to the desired height. The warm-up (two sets of 6 to 8 reps) was performed with a submaximal, nonfatiguing load for the standing calf raise. The test-retest reliability has been reported to be high, with an ICC = 0.99 [21].

The warm-up for the jump and sprint tests consisted of nonspecific running at low-to-medium intensity for approximately $5 \mathrm{~min}$. Then, coordination exercises, such as running with lifted knees, heeling, and side stepping, were performed for approximately $5 \mathrm{~min}$. Subsequently, 3 acceleration runs over approximately $30 \mathrm{~m}$ were performed with short intervening walking breaks. Overall, the total warm-up time on each test day was 15 min. To assess sprint performance, the 20-m LS and COD sprinting time was measured via timing gates. The COD and $20-\mathrm{m}$ LS tests were separated by a 15 min break. 20-m LS performance was measured over a distance of $20 \mathrm{~m}$. The test-retest reliability was reported to be high, with an ICC $=0.94-0.97$ [24]. Each athlete had three attempts. Between each completed sprint, the athletes rested for $3 \mathrm{~min}$. The subjects also performed three attempts for the COD test, which were separated by a 3-min break. The COD test involved an equilateral triangle design ( $60^{\circ}$ angles), and the test-retest reliability has been reported to be high, with an ICC $=0.88-0.91$ [22]. Bars were used to mark the corners of the triangle. Each side of the triangle was 5 meters long. To determine the time for a COD, subjects sprinted $2.5 \mathrm{~m}$, and a COD was then performed. After the subjects sprinted for another $5 \mathrm{~m}$, a second change in direction was required. Then, they sprinted for another $2.5 \mathrm{~m}$ through the final light gate. The total running distance was $10 \mathrm{~m}$. The CODs were performed to the right side. If the pylons or hurdle bars were knocked down or touched during COD testing, a follow-up attempt was completed. The time was measured for both sprint tests with a double-timing gate system (wk7 time watch, Ditzingen, Germany). The starting point was marked with a small cap $0.75 \mathrm{~m}$ away from the starting gate to avoid early triggering. The best trial was used for the statistical analysis.

Jumping performance was measured using a contact mat (Refitronic, Schmitten, Germany) that operates as a switch. This system sent information to a computer regarding whether the mat was loaded. From this information, the flight time and the jump height were determined for all jumps. The jump height was calculated from the flight time $\left(\mathrm{gt}^{2} / 8 ; \mathrm{g}=\right.$ the gravitational acceleration [9.81 $\mathrm{m} \cdot \mathrm{s}-2$ ] and $\mathrm{t}=$ flight time). The test-retest reliability for the jump has been reported to be high, with an ICC $=0.85-0.93[21,25]$. The subjects performed 5 trials for each jump, and their best result was used for analysis. The athletes rested for $15 \mathrm{~min}$ between every jump and for $1 \mathrm{~min}$ between each attempt. The SJ was initiated at a knee angle of $90^{\circ}$ without countermovement and arm swings. The DJ was performed from different heights $(24 \mathrm{~cm}, 32 \mathrm{~cm}$ and $40 \mathrm{~cm})$. The participants were instructed to take a horizontal step from a box. Immediately after their feet contacted the ground, the participants were asked to jump as high as possible. Their heels were not allowed to touch the ground. A shorter duration of ground CT (milliseconds [ms]) and a higher jump (millimeters [mm]) result in better short SSC performance. Hands were fixed at the hips. From these data, the reactive strength index (RSI) was calculated $(\mathrm{RSI}=$ jump height $(\mathrm{JH})$ in $\mathrm{mm} / \mathrm{CT}$ in $\mathrm{ms} \times 100)$ to quantify overall DJ performance.

\section{Analysis}

The data was analyzed using SPSS 26.0. (IBM, Ehningen, DE, Germany). The significance level for all statistical tests was set at $<0.05$. The descriptive statistics for all measures in each age group are presented as the mean \pm standard deviation (SD). The Shapiro-Wilk test was used to test the normality of the data.

Reliability analyses were performed using the ICC and a $95 \%$ confidence limit. Furthermore, one-tailed bivariate Pearson correlation for normally distributed data and Kendall's correlation analysis for nonnormally distributed data were used to assess the relationships in the results between the SJ, DJ, LS und COD and maximum strength tests.

\section{Results}

The Shapiro-Wilk test results showed that all parameters were normally distributed, except the 1RM SCR. The mean performance and SD for all the different tests and the ICC and the 95\% confidence intervals (95\% $\mathrm{Cls})$ for the performance tests are displayed in (Table 1$)$. The ICCs $(0.86-0.98)$ of the tests were greater than 0.70 , indicating a very strong to nearly perfect correlation and therefore good reliability according to Shrout and Fleiss [26].

The correlation coefficients showed a significant moderate to strong correlation of 1 RM BS with SJ, COD and LS. Nonsignificant weak to moderate coefficients 
Table 1: Reliability of the performance variables

\begin{tabular}{|l|l|l|}
\hline Fitness Test & Mean \pm SD & ICC $(95 \% \mathrm{Cl})$ \\
\hline 1RM BS & $80.5 \pm 14.8 \mathrm{~kg}$ & $0.91(0.81-0.96)$ \\
\hline REL BS & $1.29 \pm 0.18$ & Calculated from the 1 RM BS divided by body mass \\
\hline 1RM SCR & $110.8 \pm 18.7 \mathrm{~kg}$ & $0.86(0.65-0.95)$ \\
\hline REL SCR & $1.78 \pm 0.17 \mathrm{~kg}$ & Calculated from the 1 RM SCR divided by body mass \\
\hline SJ & $36.0 \pm 5.5 \mathrm{~cm}$ & $0.98(0.95-0.99)$ \\
\hline RSI 24 & $1.97 \pm 0.46$ & $0.88(0.74-0.95)$ \\
\hline RSI 32 & $1.76 \pm 0.38$ & $0.91(0.81-0.96)$ \\
\hline RSI 40 & $1.84 \pm 0.58$ & $0.88(0.74-0.95)$ \\
\hline $20-m$ LS & $3.04 \pm 0.14 \mathrm{~s}$ & $0.91(0.80-0.96)$ \\
\hline COD & $3.28 \pm 0.12 \mathrm{~s}$ & $0.86(0.65-0.95)$ \\
\hline
\end{tabular}

Mean test scores \pm SDs and reliability data for all players; ICC = intraclass correlation coefficient; $\mathrm{Cl}=$ confidence intervals; $1 \mathrm{RM}$ $\mathrm{BS}=$ one-repetition maximum for the back squat; $1 \mathrm{RM}$ SCR = one-repetition maximum for the standing calf raise; $\mathrm{SJ}=\mathrm{squat}$ jump; RSI 24 = reactive strength index from the $24 \mathrm{~cm}$ falling height; RSI 32 = reactive strength index from the $32 \mathrm{~cm}$ falling height; RSI 40 = Reactive Strength Index from the $40 \mathrm{~cm}$ falling height; $20-\mathrm{m} \mathrm{LS}=20$-meter linear sprint; COD = change of direction sprint; s $=$ seconds; $\mathrm{kg}=$ kilogram, $\mathrm{cm}=$ centimeter .

Table 2: Correlation and determination coefficients for the change of direction sprint, linear sprint, squat jump performance and maximum strength tests.

\begin{tabular}{|c|c|c|c|c|c|c|c|}
\hline Fitness Tests & & SJ & RSI 24 & RSI 32 & RSI 40 & 20-m LS & COD \\
\hline \multirow[t]{2}{*}{ 1RM BS } & $r$ & $0.70^{*}$ & 0.24 & 0.33 & 0.12 & $-0.46^{*}$ & $-0.50^{*}$ \\
\hline & $r^{2}$ & $0.49^{*}$ & 0.06 & 0.11 & 0.01 & $0.21^{*}$ & $0.25^{*}$ \\
\hline \multirow[t]{2}{*}{ REL BS } & $r$ & $0.35^{\star}$ & 0.24 & $0.42^{*}$ & $0.40^{*}$ & -0.10 & -0.28 \\
\hline & $r^{2}$ & $0.13^{*}$ & 0.06 & $0.18^{*}$ & $0.16^{*}$ & 0.01 & 0.08 \\
\hline \multirow[t]{2}{*}{ 1RM SCR } & $r_{t}$ & $0.35^{\star}$ & 0.21 & 0.20 & 0.00 & $-0.36^{*}$ & -0.24 \\
\hline & $r_{t}^{2}$ & $0.13^{*}$ & 0.04 & 0.04 & 0.00 & $0.13^{*}$ & 0.06 \\
\hline \multirow[t]{2}{*}{ REL SCR } & $r$ & -0.10 & $0.56^{*}$ & $0.41^{*}$ & $0.49^{*}$ & 0.16 & 0.15 \\
\hline & $r^{2}$ & 0.01 & $0.32^{*}$ & $0.17^{\star}$ & $0.24^{*}$ & 0.03 & 0.02 \\
\hline
\end{tabular}

$r=$ Pearson's correlation coefficient; $r_{t}=$ Kendall's tau correlation coefficient $=1$ RM BS = one-repetition maximum for the back squat; $1 \mathrm{RM} \mathrm{SCR}=$ one-repetition maximum for the standing calf raise; $\mathrm{SJ}=$ squat jump; $\mathrm{RSI} 24$ = reactive strength index from the $24 \mathrm{~cm}$ falling height; RSI 32 = reactive strength index from the $32 \mathrm{~cm}$ falling height; RSI 40 = reactive strength index from the 40 $\mathrm{cm}$ falling height; $20-\mathrm{m} \mathrm{LS}=20$-meter Linear Sprint; COD = change of direction sprint; ${ }^{*}=$ level of significance $(p<0.05)$

were found for 1RM BS and DJ performance. REL BS showed nonsignificant weak correlations for LS and COD performance and significant moderate correlation coefficients for SJ and DJ performance. The 1RM SCR showed a significant moderate correlation with only SJ and LS and a nonsignificant weak correlation with all other variables. Moderate to strong correlations were found between REL SCR and the DJ height. All correlation coefficients are displayed in (Table 2).

\section{Discussion}

This study was designed to evaluate and compare the influence of maximum strength in SCR and BS on sprinting, jumping and COD performance in youth soccer players. The results of this study show weak to strong correlations of absolute and relative BS and SCR performance with jump and sprint performance. In general, the results of this study are in line with the correlation coefficients reported in previous research and show the importance of maximum strength (BS/SCR) on SJ, 20-m LS and COD performance $[10,11,13,20]$. Considering the results of the two maximum strength tests and their correlation coefficients with jump and sprint performance, the following observation was made: The 1RM BS coefficients for the variables SJ, 20-m LS and COD are substantially higher than the coefficients of $1 \mathrm{RM}$ SCR. The reason for this result may be due to the high concordance of the muscle chains involved between the squats and movements such as the SJ. The plantar flexors contribute to the generation of the impulse to a lesser extent than do other muscle. The opposite is true for the influence on DJ performance. In addition to the muscles of the knee and hip joint, the muscles of the ankle joint play a performance-limiting role (triceps surae and tibialis anterior). These muscles play this role mainly because the knee flexion angle at ground contact is small, and the ground contact time is below $250 \mathrm{~ms}$, and therefore, the length change in the quadriceps muscles is small [27]. Therefore, Hartmann, et al. [21] found moderate to strong correlations of 1RM SCR with the DJ from different heights $\left(r^{2}=0.13-0.33\right)$, supporting Schmidtbleicher's [15] theory that maximum strength 
performance is a key factor of SSC performance, as well as the tendomuscular and reflex mechanisms [16]. However, this relationship was not found with regard to the absolute strength values in this study. This finding can be explained as follows: in Hartmann, et al.'s [21] study, the subjects showed higher performance in 1RM SCR but considerably lower performance in the DJ than in this study. The heterogeneity in DJ performance of the participants in their study may have led to higher coefficients. As an abort criterion for the 1RM SCR assessment, stabilization problems in the trunk were frequently noted in the subjects of this study. An underestimation of the 1RM SCR for the calf muscles in this study can be assumed.

Considering the correlation coefficients for the correlations between the relative and absolute strength in BS and SCR and jump and sprint performance, it is obvious that these factors affect the $\mathrm{JH}$. The correlation coefficients for the SJ, LS and COD were lower with REL BS or REL SCR but higher for the DJ at different heights. This finding could indicate that relative or absolute strength performance have different levels of importance for power performance variables. The ground reaction forces at the drop heights used in this study may be higher than those used for the SJ and sprint tests. DJs (20-60 cm drop height) with ground contact times up to $260 \mathrm{~ms}$ result in ground reaction forces of 4.2 6.3 times the body weight [28]. Ground reaction forces during the sprint start, however, are approximately only 2 - 5 times the body weight, while during the acceleration phase, they are still 2 - 3 times the body weight [29]. This finding might explain the relevance of strength performance relative to body weight regarding reactive strength output with high ground reaction forces. This finding might also imply that as the drop height increases for the DJ, the influence of the relative power output must increase. However, this idea cannot be confirmed on the basis of the data collected here because the drop heights used in this study are too small.

In principle, this study shows reliable results for the 1RM SCR. However, it was shown that the abort criterion that was met in many cases was the failure to achieve the required range of motion with a stable trunk and complete extension in the hip and knee joints. Therefore, an underestimation of the 1RM SCR and consequently of the corresponding correlation coefficients to jump and sprint must be assumed [22]. In future studies, a reduction in the degrees of freedom (free weight vs. machine-based) in measuring the 1RM SCR is therefore recommended.

\section{Conclusion}

This study shows that the 1RM BS has a moderate to strong correlation with SJ, LS and COD performance. However, performance, as measured by the RSI, independent of the DJ height, can be explained to a greater extent via the 1RM SCR. Therefore, the 1RM of the squat and standing calf raise should be included in strength tests for soccer players in the weight room. In addition, both the relative and absolute maximum strength of athletes should be considered in the diagnosis. Furthermore, these data imply that soccer players must incorporate strength training for squats and SCR into their training schedule to improve their sprint and jump performance. In general, additional investigations are needed to determine to what degree an increase in performance can be expected due to strength training.

\section{Acknowledgements}

The authors thank the athletes and coaches that supported this investigation.

\section{Supporting Agencies}

The authors state no conflicts of interest.

\section{Disclosure Statement}

No author has any financial interests or received any financial benefits from this research.

\section{References}

1. Bloomfield J, Polman R, O'Donoghue P (2007) Physical demands of different positions in FA Premier League soccer. J Sports Sci Med 6: 63-70.

2. Barnes C, Archer D, Hogg B, Bush M, Bradley P (2014) The evolution of physical and technical performance parameters in the English Premier League. Int J Sports Med 35: 10951100.

3. Wallace JL, Norton KI (2014) Evolution of world cup soccer final games 1966-2010: game structure, speed and play patterns. J Sci Med Sport 17: 223-228.

4. Reilly T, Bangsbo J, Franks A (2000) Anthropometric and physiological predispositions for elite soccer. J Sports Sci 18: $669-683$.

5. Jones P, Bampouras T,Marrin K (2009) An investigation into the physical determinants of change of direction speed. J Sports Med Phys Fitness 49: 97-104.

6. Bangsbo J, Mohr M, Krustrup P (2006) Physical and metabolic demands of training and match-play in the elite football player. J Sport Sci 24: 665-674.

7. Abe T, Dehoyos D, Pollock M, Garzarella L (2000) Time course for strength and muscle thickness changes following upper and lower body resistance training in men and women. Eur J Appl Physiol 81: 174-180.

8. Always E, Stray-Gundersen, J, Grumbt W, Gonyea W (1990) Muscle cross-sectional area and torque in resistance-trained subjects. Eur J Appl Physiol 60: 86-90.

9. Fukunaga T, Miyatani M, Tachi M, Kouzaki M, Kawakami Y, et al. (2001) Muscle volume is a major determinate of joint torque in humans. Acta Physiol Scand 172: 249-255.

10. Comfort P, Stewart A, Bloom L, Clarkson B (2014) Relationships between strength, sprint, and jump performance in well-trained youth soccer players. $J$ Strength and Cond Res 28: 173-177.

11. Hori N, Newton R, Andrews W, Kawamori N, McGuigan M, et al. (2008) Does performance of hang power clean differentiate performance of jumping, sprinting, and changing of direction? J Strength Cond Res 22: 412-418. 
12. Keiner M, Sander A, Wirth K, Hartmann H, Yaghobi D (2014) Correlations between maximal strength tests at different squat depths and sprint performance in adolescent soccer players. Am J Sports Sci 2: 1-7.

13. Wisloff U, Castagna C, Helgerud J, Jones R, Hoff J (2004) Strong correlation of maximal squat strength with sprint performance and vertical jump height in elite soccer players. $\mathrm{Br}$ J Sport Med 38: 285-288.

14. Broich H (2009) Quantitative methods for performance diagnostics. Cologne: German Sport University Cologne 4052.

15. Schmidtbleicher D (1992) Training for power events. In: P Komi, Strength and power in sport. Boston: Blackwell Scientific Publications 381-395.

16. Finni T, Ikegawa S., Lepola V, Komi P (2003) Comparison of force-velocity relationships of vastus lateralis muscle in isokinetic and in stretch-shortening cycle exercises. Acta Physiol Scand 177: 483-491.

17. Ferreira L, Weiss L, Hammond K, Schilling B (2010) Structural and functional predictors of drop vertical jump. J Strength Cond Res 24: 2456-2467.

18. Dymond C, Flanagan EP, Turner AP (2011) The relationship between maximal-strength and plyometric ability in Rugby players. Port J Sport Sci 11: 77-80.

19. Beattie K, Carson BP, Lyons M, Kenny IC (2017) The relationship between maximal-strength and reactive-strength. Int J Sports Physiol Perform 12: 548-553.

20. Möck S, Hartmann R, Wirth K, Rosenkranz G, Mickel C (2018) Correlation of dynamic strength in the standing calf raise with sprinting performance in consecutive sections up to 30 meters. Res Sports Med 26: 474-481.

21. Hartmann R, Möck S, Wirth K, Mickel C (2017) Relationship between the dynamic maximum strength of the plantar flex- ors and the reactive strength performance in the drop jump from different heights. Leipziger sportwiss Beitr 58: 41-58.

22. Keiner M, Sander A, Wirth K, Schmidtbleicher D (2013) Long-term strength training effects on change-of-direction sprint performance. J Strength Cond Res 28: 223-231.

23. Wirth K, Hartmann H, Sander A, Mickel C, Szilvas E, et al. (2016) The impact of back squat and leg-press exercises on maximal strength and speed-strength parameters. J Strength Cond Res 30: 1205-1212.

24. Sander A, Keiner M, Wirth K, Schmidtbleicher D (2013) Influence of a 2-year strength training programme on power performance in elite youth soccer players. Eur J Sport Sci 13: $445-451$.

25. Keiner M, Sander A, Hartmann H, Mickel C, Wirth K (2018) Do long-term strength training and age affect the performance of drop jump in adolescents? Austr J Strength Cond 26: 24-38.

26. Shrout PE, Fleiss JL (1979) Intraclass correlations: uses in assessing rater reliability. Psychol Bull 86: 420-428.

27. Schmidtbleicher D, Gollhofer A (1982) Neuromuscular studies to determine individual load parameters for deep jump training. Leistungssport 12: 298-307.

28. Ball NB, Stock CG, Scurr JC (2010) Bilateral contact ground reaction forces and contact times during plyometric drop jumping. J Strength Cond Res 24: 2762-2769.

29. Lafortune MA, Valiant GA, McLean B (2000) Biomechanics of running. In: JA Hawley (Edn), handbook of sports medicine and science - running. Oxford: Blackwell Sciences 2843. 